\title{
Control inadecuado del dolor agudo postoperatorio: prevalencia, prevención y consecuencias. Revisión de la situación en Latinoamérica
}

\section{Inadequate management of acute postoperative pain: prevalence, prevention, and consequences. Review of the situation in Latin America}

Dra. Patricia Abella-Palacios, ${ }^{*}$ Dr. Fernando Arias-Amézquita, ${ }^{\ddagger}$ Dr. Augusto R Barsella, ${ }^{\S}$
Dra. Berenice C Hernández-Porras, ${ }^{\natural}$ Dr. Douglas Kenji Narazaki,
Dra. Patricia A Salomón-Molina, ${ }^{* *}$ David Herrero-Martín Ph.D., ${ }^{\ddagger}$ Dra. Rocío Guillén-Núñez ${ }^{\S \S}$

Citar como: Abella-Palacios P, Arias-Amézquita F, Barsella AR, Hernández-Porras BC, Narazaki DK, Salomón-Molina PA, et al. Control inadecuado del dolor agudo postoperatorio: prevalencia, prevención y consecuencias. Revisión de la situación en Latinoamérica. Rev Mex Anestesiol. 2021; 44 (3): 190-199. https://dx.doi.org/10.35366/99666

RESUMEN. Introducción: El dolor postoperatorio tiene un alto impacto, es una de las principales causas médicas de retraso en el alta hospitalaria. Asimismo, es causa frecuente de readmisión hospitalaria, retrasos en la recuperación y mayores costos para el sistema de salud y los pacientes. El objetivo del presente trabajo es conocer mejor la situación del dolor agudo postoperatorio en Latinoamérica mediante una revisión bibliográfica para poder establecer su prevalencia y evaluar su magnitud. Material y métodos: Se efectuó una búsqueda bibliográfica en SciELO y PubMed tratando de recopilar la información más detallada, precisa y actualizada. Resultados: En Latinoamérica la falta de políticas claras para la evaluación y el tratamiento del dolor postoperatorio, así como de formación, conduce a un control inadecuado del mismo con una prevalencia de dolor agudo postoperatorio moderado/severo superior a 40\%. Conclusiones: El manejo del dolor agudo postoperatorio continúa siendo un problema en Latinoamérica. Muchos pacientes refieren dolor moderado o severo tras la cirugía, lo que puede conducir a dolor crónico. Se necesitan más estudios al respecto para poder establecer aún con mayor precisión la prevalencia del dolor agudo postoperatorio y los efectos derivados de su pobre control.

ABSTRACT. Introduction: Postoperative pain has a profound impact. It is one of the main causes of delayed hospital discharge and it is associated with hospital readmission, recovery problems, and higher costs both for the healthcare system and the patients. The aim of this work is to shed light on the postoperative acute pain management in Latin America through a review of the literature, in order to have a better understanding of its prevalence and the extent of the problem. Material and methods: A bibliographical search was performed in SciELO and PubMed trying to gather the most precise, detailed and updated information. Results: In Latin America, the absence of clear policies for the evaluation and treatment of postoperative pain, as well as the lack of training, leads to its inadequate control with a prevalence of moderate/severe acute postoperative pain greater than 40\%. Conclusions: Postoperative acute pain continues to be a problem in Latin America. Many patients still suffer moderate to severe pain after surgery, leading to a chronic or persistent painful condition. More studies are needed to get a clear picture of the prevalence of acute postoperative pain and the deleterious effects of an inadequate management.

\section{INTRODUCCIÓN}

A nivel mundial se realizan alrededor de 313 millones de cirugías al año y en gran parte de ellas los pacientes sufren dolor postoperatorio ${ }^{(1)}$. Una buena definición de éste es la dada por la American Society of Anesthesiologists (ASA) como la sensación presente en el paciente operado debido a la enfermedad, al procedimiento quirúrgico y sus complicaciones, o a la combinación de ambos. Se trata de un dolor que es predecible, al ser el resultado de una

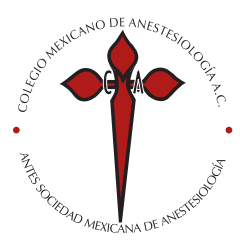

Palabras clave: Dolor postoperatorio, prevención, prevalencia, control, Latinoamérica.

Keywords: Postoperative pain, prevention, prevalence, pain management, Latin America.

* Clínica de Dolor, Hospital el Tunal. Bogotá, Colombia.

‡ Universidad de los Andes. Fundación

Santa Fe de Bogotá. Bogotá, Colombia.

$\S$ Grupo de Dor Unidade Ifor,

Rede D’or, São Caetano do

Sul, São Paulo, Brasil.

" Instituto Nacional de Cancerología, Ciudad de México, México.

" Hospital Sírio-Libanês,

São Paulo, Brasil.

** Pfizer Inc. Bosques de

las Lomas, México.

抹ACINDES (Asociación Civil de Investigación y Desarrollo en Salud) Internacional. Madrid, España. $\S \S$ Clínica del Dolor, Instituto Nacional de Cancerología (INCan). Ciudad de México, México.

Correspondencia:

Dra. Rocío Guillén-Núñez

Clínica del Dolor y Cuidados

Paliativos del Instituto Nacional

de Cancerología (INCan),

Av. San Fernando Núm. 22,

Col. Belisario Domínguez Secc.

16, 14080 Alcaldía Tlalpan,

Ciudad de México, México.

Abreviaturas:

ASA $=$ American Society

of Anesthesiologists.

ERAS = Enhanced Recovery

After Surgery.

EVA = Escala visual análoga.

ENA = Escuela numérica análoga.

$\mathrm{EVN}=$ Escala visual numérica.

PCA = Analgesia controlada por el paciente.

PROSPECT $=$ Procedure-Specific Postoperative Pain Management.

IASP = International Association for the Study of Pain.

AINE $=$ Antiinflamatorios no esteroideos.

DCPQ = Dolor crónico

postquirúrgico.

Recibido: 25-09-2019

Aceptado: 21-10-2020 
«agresión» planificada y deliberada al cuerpo del paciente, que aparece al inicio de la intervención quirúrgica y que termina, o debería hacerlo, con la curación de la enfermedad que lo ha originado ${ }^{(2,3)}$.

Dado el gran impacto negativo del dolor postoperatorio en el paciente es muy importante aliviarlo de cara a promover una curación y rehabilitación efectiva ${ }^{(4)}$. El dolor postoperatorio incrementa la morbilidad, la mortalidad y prolonga la estancia hospitalaria ${ }^{(5)}$; es fuente de importantes y numerosos retrasos en el alta hospitalaria, así como también es motivo de readmisión de un buen número de pacientes y dio origen hace 20 años a los programas denominados «Enhanced Recovery After Surgery» (ERAS) ${ }^{(6)}$. Además, es considerado un problema de salud y un tema de gran importancia a nivel mundial.

Al tener en cuenta los efectos sobre los pacientes, los limitados recursos de los sistemas de salud de buena parte de los países de Latinoamérica y dado que se estima que las operaciones quirúrgicas aumentarán en los próximos años ${ }^{(5,7)}$ se entiende la gravedad del problema que enfrentamos.

De acuerdo con el Instituto de Medicina de Estados Unidos, $80 \%$ de los pacientes refieren dolor tras una cirugía y $88 \%$ de ellos lo califican como moderado, severo o extremo ${ }^{(8)}$. En Latinoamérica no se ha realizado un estudio epidemiológico global sobre la prevalencia del dolor agudo postoperatorio ${ }^{(9)}$. Los pocos estudios que existen tratan sobre las realidades a nivel local de un centro sanitario o nacional a lo sumo ${ }^{(10-12)}$. Todo esto dificulta notablemente efectuar una evaluación real de la magnitud del problema.

A pesar del mayor conocimiento en las últimas décadas de los mecanismos que originan el dolor postoperatorio, así como de las recomendaciones de guías clínicas y los nuevos procedimientos técnicos ${ }^{(13)}$, el inadecuado control del mismo continúa siendo un problema de salud no resuelto. Por tanto, existe una necesidad urgente de mejorar el tratamiento del dolor postoperatorio, iniciando por el conocimiento de la prevalencia y el impacto en el paciente junto a las posibles medidas de prevención ${ }^{(9)}$. Para ello es fundamental tener una imagen real y actualizada de la situación del dolor postoperatorio en Latinoamérica y ese es el objetivo de la presente revisión.

\section{MATERIAL Y MÉTODOS}

Esta revisión narrativa partió de una búsqueda bibliográfica en la literatura médica y científica tanto en inglés como en español en las bases de datos SciELO y PubMed. La búsqueda se llevó a cabo durante los meses de mayo y junio de 2020 y se trató que fuera lo más amplia, profunda y actualizada posible de todos aquellos artículos relacionados con dolor postoperatorio en general y específicamente en Latinoamérica. De los estudios incluidos se recolectó información relacionada con la prevalencia, el impacto, el manejo y las medidas preventivas.
La revisión está planteada como las respuestas a una serie de preguntas sobre el tema.

\section{¿CUÁL ES LA SITUACIÓN ACTUAL DEL MANEJO DEL DOLOR POSTOPERATORIO EN LATINOAMÉRICA? EPIDEMIOLOGÍA}

Tal y como hemos comentado en la introducción no existe un estudio epidemiológico global en el conjunto de países latinoamericanos acerca de la prevalencia del dolor postoperatorio como se ha realizado en EUA ${ }^{(14,15)}$ o Europa ${ }^{(16,17)}$. Los distintos estudios publicados exponen la experiencia de una clínica en particular o de un país en el mejor de los casos. En 2017, Santos García y colaboradores publicaron un estudio que recogía los artículos publicados hasta aquel momento ${ }^{(9)}$. En los últimos tres años han aparecido nuevos estudios, algunos de los cuales reunieron una parte de los citados en la revisión mencionada ${ }^{(9)}$ y algunos otros aparecen reflejados en la Tabla 1. A continuación los detallaremos por país:

\section{Brasil}

En 2012 se llevó a cabo en cinco unidades de ingreso de un hospital (Universidade Federal do Triangulo Mineiro, Uberaba) un estudio exploratorio, descriptivo y transversal con el objetivo de determinar, entre otros parámetros, la prevalencia del dolor postquirúrgico. La tasa de prevalencia hallada fue de $48 \%{ }^{(12)}$.

Dentro de otro estudio similar en el Hospital Barao de Lucena (Recife), la prevalencia del dolor postoperatorio fue de $46 \%$ en las primeras 24 horas tras la operación, con una intensidad moderada o severa en $70.6 \%$ de ellos ${ }^{(18)}$.

En otra publicación muy reciente con 50 pacientes operados de escoliosis neuromuscular, en un hospital de tercer nivel en São Paulo, en el período comprendido entre enero y julio de 2017, $42 \%$ de los mismos manifestaron dolor moderado o severo ${ }^{(19)}$.

El dolor postoperatorio afecta a pacientes sometidos a todo tipo de cirugías, como por ejemplo la cardíaca por esternotomía. Un estudio prospectivo de una cohorte de 48 pacientes de la Irmandade da Santa Casa de Misericórdia (São Carlos) mostró que $29 \%$ padecía dolor leve/moderado en reposo y el porcentaje se incrementaba hasta $83 \%$ cuando al toser manifestaban dolor moderado o severo, $85 \%$ al girarse o en $79 \%$ al respirar profundamente. Salvo en lo relativo a la tos, a medida que pasan los días la tasa de incidencia del dolor postoperatorio disminuye, aun cuando es estadísticamente significativa $^{(20)}$.

\section{Colombia}

Un estudio multicéntrico, en siete ciudades, en el que se evaluó a un total de 1,015 pacientes mayores de 18 años, concluyó que 
la intensidad del dolor no se logró controlar adecuadamente en 600 pacientes de esa muestra (59.1\%) ${ }^{(21)}$.

En otro estudio con 153 pacientes del Hospital Universitario San Jorge de Pereira se observó que $38.8 \%$ de los pacientes no tenía controlado el dolor 24 horas después de la cirugía. La tasa aumentaba hasta $49.2 \%$ en los casos de cirugía ortopédica y curiosamente ninguno de los 11 pacientes analizados que se sometieron a un procedimiento urológico refirió dolor ${ }^{(22)}$.

En otro ensayo realizado en el Hospital Universitario San Vicente de Paúl de Medellín se identificó que 31.3\% de los pacientes postoperados presentaban dolor moderado en reposo y $22.3 \%$ severo $^{(11)}$.

Otro estudio de corte transversal evaluó la percepción del dolor a las cuatro horas de la operación en los pacientes del Hospital Universitario San Jorge de Pereira y encontró que $51.4 \%$ de los pacientes no lo tenía controlado y $30.6 \%$ relataron sufrir un dolor severo o muy severo ${ }^{(23)}$.

\section{Chile}

Un estudio publicado en 2013 examinó a 923 pacientes del programa de dolor agudo de la Clínica Alemana de Santiago entre diciembre de 2010 y septiembre de 2011. El 93\% de ellos presentó dolor postoperatorio que fue moderado o severo en $59 \%$ de los mismos ${ }^{(24)}$.

\section{México}

Este es uno de los países de Latinoamérica con un mayor número de estudios. En 2012 se realizó uno con 418 pacientes de la Unidad Médica de Alta Especialidad «Dr. Victorio de la Fuente Narváez» del IMSS, en la Ciudad de México, en el que se observó una prevalencia de dolor postoperatorio de moderado a severo de 85.2 y $79.5 \%$ a las 24 y 72 horas, respectivamente. En cuanto al dolor promedio se hizo de acuerdo con la clasificación EVA ([escala visual análoga], sin dolor con un valor de 0 , dolor leve de 1-4, dolor moderado de 5-6 y dolor severo de 7-10). El valor de EVA al ingreso fue de $5.33 \pm 2.33$ y por desgracia no varió apenas, siendo de 5.97 \pm 2.04 en el postquirúrgico inmediato ( 24 horas) y de $5.4 \pm$ 2.02 en el tardío (72 horas) ${ }^{(25)}$.

En otro estudio llevado a cabo en el Instituto Nacional de Ciencias Médicas y Nutrición «Salvador Zubirán», 63.9\% de los pacientes tuvieron dolor de moderado a severo entre las seis y 24 horas posteriores al procedimiento quirúrgico. La media de la máxima intensidad del dolor medido por la ENA (escala numérica análoga) y la EVN (escala visual numérica) en las primeras 24 horas fue de $4.98 \pm 3.1$, mientras que las cirugías con mayor intensidad de dolor reportado fueron la ortopédica (ENA $6.4 \pm 3.1$ ), la torácica (ENA $6.2 \pm 2.1$ ), la ginecológica (ENA $5.6 \pm 3.0$ ) y la laparoscópica (EVN 5.5 $\pm 3.1)^{(26)}$.

Con relación a las cirugías ginecológicas, una publicación de 2012 refirió una incidencia de dolor postoperatorio de 94.7\%. La histerectomía fue la intervención realizada con mayor frecuencia del conjunto y la media de dolor de la EVN fue de $4.2 \pm 2.08$ puntos a las $24-36$ horas $^{(27)}$.

$\mathrm{Al}$ evaluar la atención del dolor en el período postoperatorio inmediato con un estudio observacional, descriptivo, prospectivo y transversal con 180 pacientes adultos de cirugía

Tabla 1: Estudios realizados en Latinoamérica sobre el problema del dolor postoperatorio en centros de salud de la región.

\begin{tabular}{|c|c|c|c|}
\hline País & Año & Investigadores & Prevalencia del dolor postoperatorio \\
\hline \multirow[t]{5}{*}{ Brasil } & 2012 & Ribeiro SBF, et al(12) & $48 \%$ \\
\hline & 2009 & Couceiro TC, et al ${ }^{(18)}$ & $46 \%$ (moderado/severo: $70.6 \%$ de ellos) \\
\hline & 2020 & Da Silva RFF, et al ${ }^{(19)}$ & $42 \%$ (moderado/severo) \\
\hline & 2014 & De Mello LC, et al(20) & $29 \%$ (leve/moderado en reposo) \\
\hline & & & $79-85 \%$ (moderado/severo en actividades) \\
\hline \multirow[t]{4}{*}{ Colombia } & 2016 & Machado-Alba JE, et al(21) & $59.1 \%$ \\
\hline & 2013 & Machado-Alba JE, et al(22) & $38.8 \%$ (49.2\% en ortopedia) \\
\hline & 2007 & Cadavid-Puentes AM, et al(11) & Moderado: $31.3 \%$; severo: $22.3 \%$ \\
\hline & 2013 & Machado-Alba JE, et al (23) & Moderado: $20.8 \%$; severo o muy severo: $30.6 \%$ \\
\hline Chile & 2013 & Rico MA, et al(24) & 93\% (moderado/severo: 59\%) \\
\hline \multirow[t]{5}{*}{ México } & 2012 & García-Miranda GM, et al(25) & Moderado/severo: 85.2 y $79.5 \%$ ( 24 y 72 horas) \\
\hline & 2016 & Garduño-López AL, et al(26) & Moderado/severo: $63.9 \%$ (6-24 horas) \\
\hline & 2012 & Calderón-Estrada M, et al(27) & $94.7 \%$ (24-36 horas) \\
\hline & 2016 & Gómez Morales, et al (28) & $67.7 \%$ refirieron haber presentado dolor y $31.6 \%$ de carácter severo, 24 horas \\
\hline & 2018 & García-Ramírez, et al(29) & $66.3 \%$ (dolor modero, severo o muy severo, 24 horas) \\
\hline \multirow[t]{2}{*}{ Cuba } & 2019 & Hernández JM, et al(30) & $61.7 \%$ (dolor moderado) y $15.2 \%$ (dolor severo), 24 horas \\
\hline & 2012 & Velázquez González, et al(31) & $59.2 \%$ (dolor moderado) \\
\hline Costa Rica & 1998 & Sáenz-Campos $D$, et al ${ }^{(32)}$ & $38.5 \%$ con dolor moderado y $18.6 \%$ severo o muy severo, 24 horas \\
\hline \multirow[t]{2}{*}{ Uruguay } & 2006 & Saralegui J, et al $\mathrm{l}^{(33)}$ & $33 \%$ de dolor moderado/severo, pero 24 horas después ese porcentaje había bajado a 14\% \\
\hline & 2011 & Ayala S, et al(34) & $27.5 \%$ (dolor moderado/severo) \\
\hline
\end{tabular}


no ambulatoria con ASA I y II en una Unidad Médica de Alta Especialidad del IMSS (León, Guanajuato) 67.7\% refirieron haber presentado dolor y 31.6\% fue de carácter severo a las 24 horas. La variación de la ENA en este caso fue considerable desde 6.37 la máxima puntuación hasta 1.27 la mínima ${ }^{(28)}$.

Por último, cabe mencionar que en un trabajo reciente con 175 pacientes de Cirugía General, Traumatología, Ortopedia y Obstetricia/Ginecología de un Hospital de Ciudad Obregón, la prevalencia del dolor postoperatorio moderado, severo o muy severo a las 24 horas fue de $66.3 \%^{(29)}$.

\section{Cuba y Perú}

En ocasiones los estudios son conjuntos, como el del Centro Nacional de Cirugía de Mínimo Acceso de la Universidad de Ciencias Médicas de La Habana y de la Clínica Avendaño en Lima, Perú. El análisis de 298 pacientes con hiperhidrosis palmar primaria encontró dolor moderado en $61.7 \%$ de pacientes y severo en $15.2 \%{ }^{(30)}$.

Un estudio ya propiamente cubano encontró una prevalencia de $59.2 \%$ en 138 pacientes mayores de 18 años sometidos a cirugía en el hemiabdomen superior y el tórax. Dicho estudio se hizo en el Hospital Clínico Quirúrgico Hermanos Ameijeiras (La Habana) entre mayo de 2009 a mayo de 2010 (31).

\section{Costa Rica}

La prevalencia reportada fue parecida a la de otras naciones latinoamericanas. Un estudio de 1998 refería 80.5\% de pacientes con dolor (38.5\% con moderado y $18.6 \%$ severo o muy severo) a las 24 horas de haber sido sometidos a una cirugía abdominal $\operatorname{abierta}^{(32)}$.

\section{Uruguay}

Por último comentaremos la situación de Uruguay, que es otro país en el que se ha estudiado la prevalencia del dolor postoperatorio y que presenta una realidad diferente. El manejo del mismo que describen los distintos estudios refleja mejores datos y menores índices de prevalencia. Así un estudio analizó a 617 pacientes quirúrgicos del Hospital de Clínicas tratados en su Unidad de Dolor Agudo de julio de 2004 hasta enero de 2005. La tasa de dolor postoperatorio tras la cirugía era de $33 \%$ (dolor moderado o severo), pero 24 horas después ese porcentaje era de $14 \%{ }^{(33)}$.

Otro estudio que confirmó una mejoría en el control del dolor se efectuó en 2011 con 206 pacientes de la Unidad de Cirugía de día del sanatorio Centro de Asistencia del Sindicato Médico del Uruguay-Instituto de Asistencia Médica Privada Profesional (CASMU-IAMPP) mediante encuestas telefónicas a las 24 horas de la operación. El 67.2\% de los operados relató algún tipo de dolor y de ellos, en $27.5 \%$ el dolor fue percibido como moderado o severo. Un dato importante fue el alto índice de satisfacción global (93.4\% de los encuestados) $)^{(34)}$.

\section{CAUSAS DEL POBRE MANEJO DEL DOLOR POSTOPERATORIO EN LATINOAMÉRICA Y MEDIDAS PREVENTIVAS PARA MINIMIZAR SU IMPACTO}

El inadecuado control del dolor en el paciente en general y especialmente del postquirúrgico está sobre la mesa en distintos centros sanitarios mundiales (incluyendo a los latinoamericanos desde hace décadas), pese a la implementación en el uso de dispositivos de analgesia controlada por el paciente (PCA) ${ }^{(35)}$ o a la instauración de los primeros estándares y guías como la «Achieving Improved Measurement» del Canadian Council on Health Services Accreditation ${ }^{(36)}$, o la creación paulatina de unidades especializadas en dolor en cada centro hospitalario ${ }^{(37)}$. Unidades con instalaciones adecuadas, equipos multidisciplinares (médicos, cirujanos, enfermeros), estrategias propias y recursos de toda índole tales como registros clínicos ${ }^{(38)}$, nuevas terapias y programas de rehabilitación ${ }^{(39)}$ o grupos de trabajo como PROSPECT (Procedure-Specific Postoperative Pain Management), que brinda a los profesionales de la salud recomendaciones prácticas y específicas por procedimientos para facilitar la toma de decisiones en todas las etapas del período operatorio ${ }^{(40)}$, también forman parte de los intentos por mejorar el control del dolor en el terreno postoperatorio. Estos grupos han establecido protocolos específicos que aparecen en una página web (www.postoppain.org). Incluso, en los últimos tiempos, se han ido incorporando dispositivos novedosos de control y seguimiento para un correcto manejo del dolor postoperatorio $^{(41)}$. Asimismo, ha sido fundamental un mejor conocimiento de las bases moleculares y fisiológicas del dolor agudo postoperatorio $^{(42)}$. No profundizaremos en este aspecto, ya que no es objeto de este trabajo y ya existen extensas revisiones ${ }^{(43)}$.

Con relación a la principal causa del control deficiente del dolor postoperatorio, se asocia con frecuencia a un entrenamiento insuficiente del profesional sanitario desde el anestesiólogo hasta el cirujano, pasando por el personal de enfermería. Factores como la aplicación de metodologías incorrectas, fallos de organización o de recursos acaban dando pobres resultados en el manejo del dolor. De ahí la gran importancia de contar con el entrenamiento adecuado y con unidades de dolor especializadas que permitan aplicar medidas preventivas y de corrección ${ }^{(44)}$ para lograr buenos resultados, como es el caso de los centros sanitarios uruguayos mencionados ${ }^{(33,34,37)}$. Desde los 80 se ha demostrado que las unidades de dolor son la mejor opción para controlar este síndrome en los pacientes operados $^{(45)}$, no tanto por el desarrollo de técnicas novedosas, sino por la implementación de forma organizada ${ }^{(46)}$ de los 
protocolos y guías existentes ${ }^{(47)}$. Algo muy importante es que estas medidas se pueden implementar de forma eficaz en situaciones de escasez de recursos como puede suceder por desgracia en algunas de las regiones de Latinoamérica. Al utilizar los servicios de anestesia y enfermería ya existentes se puede lograr una buena atención al dolor postoperatorio en estas unidades maximizando así los recursos al alcance ${ }^{(48)}$.

Disponer de una unidad de dolor agudo nos permite monitorizar aspectos clave para su control. La valoración preoperatoria incluyendo la anestesia, el diseño e implementación del plan terapéutico previsto, la valoración de forma regular del dolor en todos los enfermos e implementar y controlar la aplicación de diferentes métodos analgésicos son tareas muy importantes, pero sobre todo la reevaluación de los resultados para hacer los ajustes necesarios y el registro sistemático en ficheros clínicos son fundamentales para un correcto abordaje del dolor postoperatorio ${ }^{(39)}$.

Otro buen ejemplo en Latinoamérica del establecimiento y el buen hacer de una unidad de dolor agudo se relata en la experiencia de la Unidad de Terapia Quirúrgica Central del Hospital General de México ${ }^{(49)}$. La clave del éxito en ese caso definido por su propia autora radica en la planificación detallada, la protocolización de los procedimientos y una estricta preparación del personal médico y de enfermería. Para poner en funcionamiento esa unidad observaron requisitos como la cobertura de 24 horas con capacidad de respuesta inmediata y la aceptación plena de la responsabilidad en el control del dolor del paciente mientras esté en la unidad. De esa forma, y mediante la introducción de un programa de analgesia multimodal y reducción del estrés, lograron reducir el número de pacientes que requirieron una estancia en la unidad de cuidados intensivos en el período inmediato tras cirugías mayores tal y como se ha demostrado en otras experiencias $^{(50)}$. En el caso concreto de este hospital mexicano el estudio se realizó con 100 pacientes de distintos tipos de cirugías (55\% en la región abdominal, 20\% en los miembros inferiores, $15 \%$ en cabeza y cuello) a los que se suministró distintos tipos de anestesia (anestesia general balanceada en $42 \%$ de los casos). A $69 \%$ de pacientes se les administró un medicamento y a $31 \%$ restante dos; el más administrado fue ketorolaco en $31 \%$ de los pacientes, seguido de la combinación ketorolaco y tramadol en $24 \%$, y tramadol como monodroga en $19 \%$. De $33 \%$ de pacientes con dolor moderado/severo a su ingreso a la unidad, este porcentaje disminuyó a 18\% al egreso; por lo tanto, se comprueba que un tratamiento adecuado del dolor en estas unidades puede minimizar la gravedad del dolor postquirúrgico.

Otro elemento preventivo se basa en el seguimiento de las guías y protocolos existentes hoy en día. El protocolo ERAS $^{(51)}$ es un conjunto de estrategias con tratamientos perioperatorios basados en la evidencia científica con el objetivo de mejorar la recuperación funcional de los pacientes tras cirugía, reduciendo lo más posible el dolor postquirúrgico, lo cual ha dado frutos en Latinoamérica posterior a su implementación, logrando resultados notables en centros hospitalarios de todo el continente ${ }^{(52)}$. Un ejemplo que evidencia ese buen funcionamiento es el trabajo llevado a cabo en el Servicio de Cirugía General del Complejo Hospitalario «Dr. José Ignacio Baldó» (Caracas, Venezuela) entre julio y septiembre de 2012. Se trata de un estudio descriptivo en pacientes sometidos a diferentes tipos de cirugía en el que se comprobó que el grupo al cual se le aplicó el protocolo ERAS presentaba menos dolor y una estancia hospitalaria más corta que la del grupo control ${ }^{(53)}$.

Hoy en día, existe ERAS LatAm, capítulo latinoamericano de la ERAS ${ }^{\circledR}$ Society, cuya misión es facilitar la implementación completa de sus programas en los países de América del Sur y el Caribe al posibilitar el acceso a sus protocolos, los cuales están disponibles de manera gratuita (http://erassociety.org/guidelines/list-of-guidelines/). En Colombia se han adaptado estos protocolos tomando como base las recomendaciones generales y estableciendo otras específicas para las situaciones concretas del panorama médico y de acceso farmacéutico del país, lo cual ha brindado mejoría en el control del dolor postoperatorio. Esta protocolización de la atención en el terreno perioperatorio permite que al ser implementadas por los prestadores de salud tengan un impacto positivo en el paciente, favorezcan el desempeño de los profesionales sanitarios y mejoren el aprovechamiento de los recursos ${ }^{(54)}$. Un ejemplo interesante al respecto ha sido puesto de manifiesto por Garduño-López y su equipo ${ }^{(55)}$ en México, que además proponen recomendaciones preoperatorias a partir de las indicaciones del grupo PROSPECT.

Otro punto importantísimo, como ya se ha citado, es el de la formación. Al tener en cuenta que en la actualidad existen suficientes estudios y opciones terapéuticas para un adecuado manejo del dolor postoperatorio quizás el problema esté en la propia concepción del dolor agudo postoperatorio y en la falta de formación sobre cómo controlarlo. De acuerdo con la International Association for the Study of Pain (IASP), en una encuesta realizada a médicos en 2005 , alrededor de $90 \%$ respondió que la educación médica sobre dolor antes de graduarse era inadecuada e incompleta ${ }^{(56)}$. Derivada de la información obtenida en este proyecto, se lanzaron una serie de iniciativas con el fin de mejorar esos fallos formativos en numerosos países, incluyendo a los de Latinoamérica ${ }^{(57)}$. Un buen ejemplo de estas iniciativas es el caso de la publicación del manual de rutas clínicas de dolor en los años 2015 y 2019 en Bogotá, Colombia, con el fin de educar a la población médica del distrito capitalino en diferentes temas de dolor agudo y crónico. Estas publicaciones han servido de apoyo para el aprendizaje de los médicos que trabajan en salas de cirugía y en otras áreas de la medicina, por fortuna cada día cobra más importancia esta formación a nivel médico en toda Colombia, lo cual cubre el hueco existente de falta de 
conocimientos acerca del control del dolor en la mayoría de programas académicos universitarios ${ }^{(58,59)}$. Pese a programas como éste, los problemas persisten como pone de manifiesto la evaluación de la capacitación de doctores y enfermeros en este tema. Un estudio con 30 doctores y 30 enfermeros reveló que si bien $63.3 \%$ del personal de enfermería conocía las escalas de dolor, tan sólo 16.6\% era consciente de la existencia de protocolos analgésicos. En el caso de los doctores los porcentajes eran similares (70 y 13.3\%) y únicamente 3.3\% de los mismos tenía formación especializada en dolor ${ }^{(60)}$. Un entrenamiento de forma continua podría contribuir a mejorar esos déficits formativos. Un programa de formación asociado a un registro o ficha de evaluación demostró su utilidad para identificar mejor el dolor e influir en el uso de medicación recetada a los pacientes de cirugía cardíaca. El objetivo final en la reducción de la intensidad del dolor del paciente se logró(61).

Lógicamente los avances farmacológicos y tecnológicos permiten obtener una mejor planificación y ejecución del proceso anestésico-quirúrgico, lo que conlleva a menores puntuaciones en la intensidad del dolor. Ejemplo de ello son los procedimientos usados en el programa denominado «Fast track» o rehabilitación multimodal para la cirugía de resección de colon y recto superior en el Hospital Militar de Santiago de Chile, el cual es un conjunto de técnicas que permiten optimizar la recuperación con una disminución en la estancia media hospitalaria, así como un decremento importante del dolor medido por EVA con valores menores a dos a las 24 horas en 95\% de los pacientes y un grado de satisfacción alrededor de $70 \%{ }^{(62)}$. En la última década este programa en el abordaje laparoscópico en distintas operaciones, como las de las patologías hematológicas ${ }^{(63)} \mathrm{o}$ las nefrectomías ${ }^{(64)}$, ha confirmado sus ventajas en el número de días de hospitalización y en menores puntaciones en dolor postoperatorio.

Dentro de las estrategias farmacológicas de tratamiento recomendadas para prevenir el dolor postoperatorio se encuentra la implementación de la analgesia preventiva. Sin entrar en el detalle concreto de las numerosas opciones analgésicas disponibles, cabe destacar dos aspectos claves para un abordaje correcto: por un lado, evaluar de forma periódica la efectividad de los tratamientos y tipos de analgesia ${ }^{(65)}$, y por otro impulsar ensayos clínicos que prueben nuevas aproximaciones. Con respecto a esto, Cuba es uno de los países con mayores investigaciones comparando la eficacia de los distintos tratamientos farmacológicos administrados de forma preoperatoria o en el postquirúrgico inmediato: morfina ${ }^{(66-68)}$, ketamina $^{(69,70)}$, ketorolaco $^{(71)}$. Un estudio descriptivo, exploratorio y retrospectivo de 2009 con 260 pacientes menores a 60 años sometidos a hemorroidectomía analizó la prevalencia de las combinaciones farmacológicas e interacciones medicamentosas de las diferentes terapias analgésicas y su asociación con los eventos adversos, identificando que en general la mayoría de los tratamientos eran eficaces con un buen perfil de seguridad ${ }^{(72)}$. Otro estudio demostró que el bloqueo bilateral del plexo cervical superficial mejoraba la analgesia en cirugía de tiroides, lo que permite disminuir el consumo de opioides y mejorar el postoperatorio de esta cirugía $^{(73)}$. La cirugía maxilar y bucal ha sido un campo prolífico para la realización de estudios, profundizando en aspectos como el uso de los antiinflamatorios no esteroideos (AINE) ${ }^{(74,75)} \mathrm{O}$ de antibióticos como la amoxicilina ${ }^{(76)}$. Otras cirugías como las de urgencia en el campo de la obstetricia ${ }^{(77)}$ o la cirugía abdominal $^{(78)}$, o las necesidades especiales en la población geriátrica $^{(79,80)}$ también han sido objeto de investigación. En cuanto a los ensayos clínicos alguno ya ha tenido lugar. Así un estudio aleatorizado, doble ciego (\# NCT02145975) verificó que no existían diferencias en cuanto al uso de morfina o fentanilo para tratar el dolor agudo postoperatorio en una unidad del dolor del Hospital San Vicente de Medellín (Colombia) (81). Otro estudio realizado en el Hospital Docente Clínico Quirúrgico «Dr. Salvador Allende», de la Habana, evaluó el uso de la analgesia postoperatoria con morfina intratecal en cirugía proctológica. Este estudio experimental prospectivo, comparativo y doble ciego permitió conocer la dosis óptima intratecal de morfina para un menor dolor postoperatorio ${ }^{(82)}$.

Un último punto a considerar es el consultar a los pacientes para evaluar su satisfacción y corregir aquellos errores que se estén cometiendo, pero no únicamente sobre el postoperatorio $^{(11)}$, sino también sobre el preoperatorio, ya que el estado del paciente puede influir de forma notable en el resultado de la cirugía. Un artículo recién publicado realizado en un Hospital de Minas Gerais (Brasil), con 65 pacientes operados del tracto digestivo, analizó la relación entre el estado emocional preoperatorio y la intensidad del dolor postoperatorio para poder establecer predictores de ese dolor. El estudio pudo identificar a la ansiedad preoperatoria como factor predictivo de riesgo para dolor postoperatorio agudo, pero no para depresión y por tanto no puede ser considerado un factor predictivo de dolor postoperatorio $^{(83)}$. Resultados similares en cuanto a la ansiedad se habían ya descrito anteriormente en España en pacientes adultos sometidos a cirugía de traumatología y ortopedia ${ }^{(84)}$. Con respecto al dolor postquirúrgico en una experiencia reciente de la Unidad de Cuidados Postanestésicos del Hospital Universitario de la Samaritana de Bogotá con 379 pacientes y usando la escala CdR se comprobó que sólo 7.14\% de los pacientes estaban satisfechos con la calidad de la recuperación ${ }^{(85)}$. La escala CdR se creó en Colombia para medir la calidad de la recuperación postanestésica desde la perspectiva de los pacientes en unidades de atención de bajo a mediano nivel de complejidad ${ }^{(86)}$. La escala CdR consta de tres dominios, entre ellos la evaluación del dolor postoperatorio; en este estudio ${ }^{(85)} \mathrm{se}$ evaluó su consistencia interna y validez en un hospital referente de alta complejidad como es el Universitario de la Samaritana de Bogotá. La escala CdR se confirmó mediante la prueba Alpha de Cronbach como un índice de consistencia interna alta, lo que pone de manifiesto que es posible desarrollar parámetros 
fiables de evaluación de la satisfacción del paciente operado adaptados a las características sanitarias locales.

\section{DISCUSIÓN}

El dolor agudo postoperatorio impacta de manera profunda en el paciente a nivel sistémico y psicológico. Manejar de forma inadecuada el dolor agudo postoperatorio trae consecuencias profundamente negativas para el paciente ${ }^{(87,88)}$. Demoras en la cicatrización de las heridas, riesgo de sufrir embolias, incremento del riesgo de infecciones postquirúrgicas, isquemias por activación simpática o problemas a nivel psicológico son algunas de esas consecuencias que motivan retrasos en la gestión del alta hospitalaria y en la recuperación del paciente ${ }^{(89,90)}$. El impacto también tiene lugar a nivel de las instituciones sanitarias afectando su desempeño, ya que aumenta la insatisfacción de los usuarios y las posibles reclamaciones y/o acciones legales, se prolonga la estancia hospitalaria con el consiguiente aumento de costos médicos y hay mayor riesgo de reingreso hospitalario ${ }^{(91,92)}$.

Los diferentes trabajos realizados arrojan datos similares en cuanto al porcentaje de pacientes que presentan dolor postquirúrgico; en la mayoría de los casos el dolor está presente en más de $30 \%$ de los pacientes y llegaba a situarse hasta por encima de $50 \%$. Curiosamente Uruguay parece ser la excepción con prevalencias por debajo de lo habitual en Latinoamérica y un alto grado de satisfacción de los pacientes en el manejo de su dolor tras sus intervenciones quirúrgicas. A pesar de las guías y los consensos existentes (en algunos casos adaptados a la realidad local) la falta de formación de los profesionales sanitarios en el tratamiento del dolor postoperatorio contribuye a su pobre control. Se necesita identificar primero esas fallas formativas con estudios de cada situación nacional para poder tomar acciones que las mejoren. Una correcta adaptación de las guías y protocolos para un manejo sencillo y claro facilitaría el correcto seguimiento de los mismos y debería acompañarse de medidas para su obligado cumplimiento al menos en cada unidad de cirugía o de cuidados postanestésicos.

Debemos evitar sobre todo que un mal manejo del dolor postoperatorio pueda derivar en un problema de salud aún mayor como es el dolor postoperatorio crónico ${ }^{(93)}$. El dolor crónico impacta en la vida cotidiana de los pacientes y afecta su salud y calidad de vida. Su incidencia global puede ser tan elevada, de hasta $50 \%$ de los pacientes operados afectados, de acuerdo con las diferentes intervenciones quirúrgicas sufridas $^{(94)}$. Su impacto económico es otro aspecto que debe tenerse en cuenta y más todavía en países con recursos de asistencia sanitaria limitados ${ }^{(95)}$. Los factores predictivos de riesgo para el desarrollo de dolor crónico postquirúrgico están claramente identificados desde hace tiempo ${ }^{(96,97)}$. Es indispensable un conocimiento preciso del dolor, sus tipos, vías y mecanismos ${ }^{(98)}$, así como de los distintos abordajes para evitar que el dolor agudo se cronifique ${ }^{(99)}$. Es muy interesante el hecho de que técnicas pensadas para su uso en dolor crónico como la ablación por radiofrecuencia o la neuromodulación pueden ayudar en el manejo del dolor postoperatorio si se aplican antes de la cirugía ortopédica ${ }^{(100)}$.

Por último, es de suma importancia recordar que en el tratamiento del dolor postoperatorio debe existir una correcta dispensación y dosificación de la analgesia opioide ${ }^{(101)}$ y más con los recientes problemas en países del continente americano como en los EUA ${ }^{(102)}$. Por eso se debe abordar el dolor postoperatorio bajo un punto de vista multifocal con distintas estrategias de prevención y tratamiento, poniendo especial cuidado en aquellos pacientes que ya reciben tratamiento opioide ${ }^{(103)}$.

\section{CONCLUSIONES}

Esta revisión ha puesto de manifiesto que si bien cada vez hay más estudios en Latinoamérica que revisan el tema específico del dolor agudo postoperatorio en países como Brasil, México o Colombia, en general hace falta disponer de más datos actualizados para toda Latinoamérica. Por lo que es necesario hacer investigaciones más profundas de sus efectos y consecuencias.

También está claro, a través de los estudios publicados, que la implementación de las clínicas de dolor con un enfoque multimodal favorece de manera importante al control del dolor. Continuar implantando nuevas técnicas quirúrgicas, la revisión periódica de los procedimientos empleados de cara a evaluar su eficacia, la realización de ensayos clínicos para probar nuevas opciones terapéuticas analgésicas, así como la constante consulta del grado de satisfacción del paciente son otros elementos clave identificados en la literatura para avanzar hacia una solución del problema.

Por lo tanto, resulta evidente que pese a diversos avances la realidad del control del dolor agudo postoperatorio en el paciente latinoamericano no es buena y que, por otra parte, es necesario realizar más estudios para disponer de bibliografía donde se profundice en la incidencia real del problema, sus consecuencias y las medidas para prevenirlo.

\section{REFERENCIAS}

1. Weiser TG, Haynes AB, Molina G, Lipsitz SR, Esquivel MM, Uribe-Leitz T, et al. Estimate of the global volume of surgery in 2012: an assessment supporting improved health outcomes. Lancet. 2015;385:S11.
2. Carr DB, Goudas LC. Acute pain. Lancet. 1999;12:2051-2058.

3. López RI, González SR, Machado GJ, De León Ortiz MA, Álvarez RD. Dolor postoperatorio agudo: el pan nuestro de cada día. Revista Archivo Médico de Camagüey. 2005;9:134-143. 
4. Tan M, Law LS, Gan TJ. Optimizing pain management to facilitate enhanced recovery after surgery pathways. Can J Anaesth. 2015;62:203-218.

5. Gan TJ. Poorly controlled postoperative pain: prevalence, consequences, and prevention. J Pain Res. 2017;10:2287-2298.

6. Kehlet H. Enhanced postoperative recovery: good from afar, but far from good? Anaesthesia. 2020;75:e54-e61.

7. Meara JG, Leather AJ, Hagander L, Alkire BC, Alonso N, Ameh EA, et al. Global Surgery 2030. Lancet. 2015;386:569-624.

8. Institute of Medicine. Relieving Pain in America: A Blueprint for Transforming Prevention, Care, Education, and Research. Washington: National Academies Press, 2011.

9. Santos GJ, Bonilla P, Campos KD, Cantú FF, Diaz P, Valtolina E, et al. Optimizing post-operative pain management in Latin America. Braz J Anesthesiol (English Edition). 2017;67:395-403.

10. Sada-Ovalle T, Delgado-Hernández E, Castellanos-Olivares A. Prevalencia del dolor postoperatorio en cirugía electiva de pacientes del hospital de especialidades del Centro Médico Nacional Siglo XXI IMSS. Rev Soc Esp Dolor. 2011;18:91-97.

11. Cadavid PA, Mendoza VJ, Gómez ÚN, Berrío VM. Prevalencia de dolor agudo postoperatorio y calidad de la recuperación en el Hospital Universitario San Vicente de Paul, Medellín, Colombia 2007. Iatreia. 2009;22:11-15.

12. Ribeiro SB, Pinto JC, Ribeiro JB, Santos FM, Martins BS, De Oliveira LF, et al. Pain management at inpatient wards of a university hospital. Braz J Anesth. 2012;62:605-611.

13. Pogatzki-Zahn EM, Segelcke D, Schug SA. Postoperative pain-from mechanisms to treatment. Pain Rep. 2017;2:e588.

14. Buvanendran A, Fiala J, Patel KA, Golden AD, Moric M, Kroin JS. The incidence and severity of postoperative pain following inpatient surgery. Pain Med. 2015;16:2277-2283.

15. Gan TJ, Habib AS, Miller T, White W, Apfelbaum JL. Incidence, patient satisfaction, perception of postsurgical pain: results from a US national survey. Curr Med Res Opin. 2014;30:149-160.

16. Fletcher D, Stamer UM, Pogatzki-Zahn E, Zaslansky R, Tanase NV, Perruchoud C, et al. Chronic postsurgical pain in Europe: an observational study. Eur J Anaesthesiol. 2015;32:725-734.

17. Benhamou D, Berti M, Brodner G, De Andres J, Draisci G, MorenoAzcoita M, et al. Postoperative Analgesic Therapy Observational Survey (PATHOS): a practice pattern study in 7 Central/Southern European countries. Pain. 2008;136:134-141.

18. Couceiro TC de M, Valenca MM, Lima LC, De Menezes TC, Raposo MCF. Prevalence and influence of gender, age, and type of surgery on postoperative pain. Rev Bras Anestesiol. 2009;59:314-320.

19. Da Silva RFF, Mendoza MAL, Pontin JCB. Epidemiological profile and outcomes in postoperative neuromuscular escoliosis. Coluna/Columna. 2020;19:26-29.

20. De Mello LC, Rosatti SFC, Hortense P. Assessment of pain during rest and during activities in the postoperative period of cardiac surgery. Rev Lat-Am Enfermagem. 2014;22:136-143.

21. Machado-Alba JE, Ramirez-Sarmiento JO, Salazar-Ocampo DF. Estudio multicéntrico sobre efectividad de control del dolor postquirúrgico en pacientes de Colombia. Rev Colomb Anestesiol. 2016;44:114-120.

22. Machado-Alba J, Machado-Duque M, Cardona F, Ruiz R. Control del dolor postquirúrgico en pacientes de un hospital de tercer nivel. Rev Med. 2013;21:46-53.

23. Machado-Alba JE, Machado-Duque ME, Calderón Flórez V, González Montoya A, Cardona Escobar F, Ruiz Garáa R, et al. ¿Estamos controlando el dolor postquirúrgico? Rev Colomb Anestesiol. 2013;41:132-138.

24. Rico MA, Veitl S, Buchuck D, Valentín HP, Subiabre D, Muñiz HC, et al. Evaluación de un programa de dolor agudo: Eficacia, seguridad y percepción de la atención por parte de los pacientes. Experiencia Clínica Alemana, Santiago-Chile. Rev Chil Anest. 2013;42:145-156.
25. García-Miranda GM, Guevara-López U, Serratos-Vázquez MC, Roa-Aguirre L, Rivera-Saldívar G. Tendencias de prescripción en el control del dolor en un Hospital de Ortopedia y Traumatología. Rev Mex Anestesiol. 2012;35:40-45.

26. Garduño-López AL, Cárdenas-Rodríguez RF, Navarrete-Pacheco M, Gutiérrez-Sougarret B, Covarrubias-Gómez A, Acosta-Nava V. Diagnóstico situacional del manejo del dolor postquirúrgico en cirugía electiva en pro de los hospitales libres de dolor. Rev Mex Anest. 2015;27:49-50.

27. Calderón-Estrada M, Pichardo-C M, Súarez-M M, RamírezMontiel M, Contreras-Carreto N. Calidad de atención del dolor postoperatorio en cirugía ginecológica. Rev Invest Med Sur Mex. 2012;19:144-148.

28. Gómez M, CE, García Posada LD, López GV. Evaluación de atención del dolor postoperatorio en la Unidad Médica de Alta Especialidad. León Guanajuato. Anest Méx. 2016;28:20-27.

29. García-Ramírez PE, González-Rodríguez, SG, Soto-Acevedo F, BritoZurita OR, Cabello-Molina R, López-Morales CM. Postoperative pain: frequency and management characterization. Rev Colomb Anestesiol. 2018;46:93-97.

30. Hernández JM, Martínez MAA, Salinas GS, Nodal JO. Estandarización de una técnica en el tiempo y su impacto en los resultados para hiperhidrosis palmar primaria. Rev Cir. 2019;71:537-544.

31. Velázquez GK, Cordero EI, Yadira OT, Valdés LR. Problemas actuales del tratamiento del dolor postoperatorio. Rev Cubana Anestesiol Reanim. 2012;11:173-184.

32. Sáenz-Campos D, Gamboa A. Control del dolor en el postoperatorio inmediato y análisis de la prescripción de analgésicos. Acta Méd Costarric. 1998;40:24-29.

33. Saralegui J, Balverde M, Baptista W, Amonte G, Lagomarsino A, Bounous A, et al. Unidad de dolor agudo postoperatorio: Casuística del Hospital de Clínicas. Rev Méd Urug. 2006;22:66-72.

34. Ayala S, Cristiani F, Saralegui J. Satisfacción del paciente en un servicio de anestesia ambulatoria. Anest Analg Reanim. 2011;24:53-58.

35. Nardi-Hiebl S, Eberhart LHJ, Gehling M, Koch T, Schlesinger T, Kranke P. Quo Vadis PCA? A review on current concepts, economic considerations, patient-related aspects, and future development with respect to patient-controlled analgesia. Anesthesiol Res Pract. 2020;2020:9201967.

36. Joint Commission on Acreditation of Healthcare Organizations. Pain assessment and management standards- Hospitals. Comprehensive Accreditation Manual for Hospitals. The Oficial Handbook (CAMH). Oakbrook Terrace IL: Joint Commission on Acreditation of Healthcare Organizations, 2001.

37. Saralegui J. Unidad de dolor agudo. Anest Analg Reanim. 2004;19:4-5.

38. Zaslansky R, Rothaug J, Chapman CR, Backstrom R, Brill S, Fletcher $\mathrm{D}$, et al. Pain out: the making of an international acute pain registry. Eur J Pain. 2015;19:490-502.

39. Mitra S, Carlyle D, Kodumudi G, Kodumudi V, Vadivelu N. New Advances in Acute Postoperative Pain Management. Curr Pain Headache Rep. 2018;22:35.

40. Lee B, Schug SA, Joshi GP, Kehlet H; PROSPECT Working Group. Procedure-Specific Pain Management (PROSPECT) - An Update. Best Pract Res Clin Anaesthesiol. 2018;32:101-111.

41. Samper BD, Barroso CP, Roca AG, Pérez HM, Monerris TM, García EL, et al. Optimization of postoperative pain management based on a new technological tool. Pain map. Rev Soc Esp Dolor. 2019;26:154-165.

42. Palada V, Kaunisto MA, Kalso E. Genetics and genomics in postoperative pain and analgesia. Curr Opin Anaesthesiol. 2018;31:569-574.

43. Gulur P, Nelli A. Persistent postoperative pain: mechanisms and modulators. Curr Opin Anaesthesiol. 2019;32:668-673.

44. Chabás E. Más retos para los anestesiólogos ¿Conseguirán las unidades de dolor agudo postoperatorio? Rev Esp Anestesiol Reanim. 2002;49:343-345. 
45. Ready LB, Oden R, Chawick HS, Benedetti C, Roock GA, Caplan R, Wild LM. Development of an Anesthesiology-based postoperative pain management Service. Anesthesiology. 1988;68:100-106.

46. Breivik H. How to implement an acute pain service. Best Pract Res Clin Anaesthesiol. 2002;16:527-547.

47. Chou R, Gordon DB, de Leon-Casasola OA, Rosenberg JM, Bickler S, Brennan T, et al. Management of postoperative pain: a clinical practice guideline from the American Pain Society, the American Society of Regional Anesthesia and Pain Medicine, and the American Society of Anesthesiologists' Committee on Regional Anesthesia, Executive Committee, and Administrative Council. J Pain. 2016;17:131-157.

48. Rawal N, Breeggre L. Organization of acute pain service: a low cost model. Pain. 1994;57:117-123.

49. Hernández-Saldívar ML. Manejo del dolor postoperatorio: experiencia terapéutica en Unidad de Terapia Quirúrgica Central del Hospital General de México. Rev Mex Anestesiol. 2008;31:246-251.

50. Rawal N. Organization, function, and implementation of acute pain service. Anesthesiology Clin N Am. 2005;23:211-225.

51. Fearon $\mathrm{KCH}$, Ljungqvist O, Von Meyenfeldt M, Revhaung A, Dejong CHC, Lassen K, et al. Enhanced recovery after surgery: a consensus review of clinical care for patients undergoing colonic resection. Clin Nutr. 2005;24:466-477.

52. McLoughlin S, Alvarez AO, Ljungqvist O. Sociedad ERAS y Latinoamérica. Rev Nutr Clin Metab. 2020;3:86-90.

53. Chalhoub BYD, Álvarez CMA, Velázquez GJ. Protocolo ERAS en pacientes sometidos a cirugía. Rev Latinoam Cir. 2013;3:5-11.

54. Calvache JA, Leon E, Gomez LM, Garcia C, Torres M, Buitrago $\mathrm{G}$, et al. Recomendaciones basadas en la evidencia del manejo de complicaciones postquirúrgicas en el contexto colombiano. Rev Colomb Anestesiol. 2015;43:51-60.

55. Garduño-López AL, Nuche-Cabrera E, Monroy-Álvarez C. Dolor postoperatorio: optimización del manejo en el contexto perioperatorio. Rev Mex Anestesiol. 2016;39:16-19.

56. Bond M, Acuna Mourin M, Barros N, et al. Education and training for pain management in developing countries: a report by the IASP Developing Countries Taskforce. Seattle: IASP Press, 2007.

57. Bond M. A decade of improvement in pain education and clinical practice in developing countries: IASP initiatives. Br J Pain. 2012;6:8184.

58. Abella P. Manual de rutas clínicas de dolor. Hospital el Tunal. Bogotá, Colombia Editorial Panamericana. 2015.

59. Abella P. 2da edición del Manual de rutas clínicas de dolor. Secretaria Distrital de Salud de Bogotá. 2019.

60. Ribeiro MCO, Pereira CU, Sallum AM, Alves JAB, Albuquerque MF, Fujishima PA. Knowledge of doctors and nurses on pain in patients undergoing craniotomy. Revista Latino-Americana de Enfermagem. 2012;20:1057-1063.

61. Silva MAS, Pimenta CAM, Cruz DALM. Treinamento e avaliação sistematizada da dor: impacto no controle da dor do pós-operatório de cirurgia cardíaca. Revista da Escola de Enfermagem da USP. 2013;47:8492.

62. Espíndola L. Cirugía de colon abierta con "Fast track" o recuperación acelerada. Rev Chilena de Cirugía. 2009;61:158-167.

63. Kim-Koh MJ, Souza-Gallardo LM. Esplenectomía laparoscópica como tratamiento para la púrpura trombocitopénica idiopática y la esplenomegalia masiva en un hospital de segundo nivel. Rev Fac Med (Méx). 2018;61:20-25.

64. López-Arias R, González-Salas M. Nefrectomía laparoscópica: impacto de su implementación. Acta Médica Costarricense. 2013;55:79-81.

65. Poggi Machuca L, Ibarra Chirinos O. Manejo del dolor agudo postquirúrgico. Acta Méd Peruana. 2007;24:39-45.

66. Correa TM, Castillo PV, García RA, Gonzáles MA, Bazart PP. Analgesia postoperatoria con morfina base en cirugía abdominal. Rev Ciencias Médicas de Pinar del Río. 2004;8:3-12.
67. Cruz DS, Ramos PL, De la Cruz SI, Campos GM. Analgesia postoperatoria con el uso de morfina liofilizada. Revista Cubana de Anestesiología y Reanimación. 2013;12:129-138.

68. Velázquez GK, Valdés LR, Pérez GY. Analgesia postoperatoria en cirugía torácica electiva. Revista Cubana de Anestesiología y Reanimación. 2010;9:107-118.

69. Hidalgo Velásquez J, Ortega Valdés ME, Morales Jiménez L, Pimienta Peguero M. Microdosis de ketamina en la prevención del dolor postoperatorio. Revista de Ciencias Médicas de Pinar del Río. 2005;9:11-20.

70. Redondo Gómez, ZA. Ketamina como medicamento coadyuvante en el control del dolor perioperatorio en la cirugía abdominal mayor. Rev Cub Anestesiol Reanim. 2013;12:210-221.

71. Granadillo G, Claudio E, Guerra M, Zúñiga M. Premedicación con ketorolac para analgesia postoperatoria en cirugía ginecológica. Archivos Venezolanos de Farmacología y Terapéutica. 2001;20:69-79.

72. Secoli SR, Moraes VC, Peniche ACG, Vattimo MFF, Duarte YAO, Mendoza IYQ. Dor pós-operatória: combinacoes analgésicas e eventos adversos. Revista da Escola de Enfermagem da USP. 2009;43:12441249.

73. Ávalos JN, Cabrera Sch C, Semertzakis PI, Schmied PS. Efectividad de anestesia de plexo cervical superficial preoperatorio para control de dolor postoperatorio en cirugía de tiroides. Rev Chil Cir. 2014;66:531535.

74. Carballosa FU, Aguilar ZG, Pacheco MC, Figueroa CL. Eficacia de la analgesia preoperatoria con antiinflamatorios no esteroidales (Aines) en cirugía de terceros molares. Revisión de la Literatura. Int J Odontostomatol. 2018;12:131-136.

75. Nícoli GA, Conte-Neto N, Campos JADB, Cabrini-Gabrielli MA, Pereira-Filho VA. Efficacy of lumiracoxib versus diclofenac sodium in pain control following extraction of impacted lower third molar. Int J Odontostomatol. 2017;11:47-51.

76. Sillet M, Orellana A, Salazar E. Estudio comparativo de tres regímenes de tratamiento Profiláctico antes de la cirugía bucal. Acta Odontológica Venezolana. 2009;47:78-91.

77. Vasallo Comendeiro VJ, Arjona Fonseca S. Analgesia multimodal postoperatoria en cirugía ginecológica y obstétrica urgente. Rev Cubana de Anestesiol Reanim. 2011;10:205-212.

78. Muñoz CM. Analgesia preventiva con drogas antiinflamatorias no esteroideas en procedimientos quirúrgicos abdominales de urgencia. Rev Cubana de Anestesiol Reanim. 2013;12:222-230.

79. Dávila CV, Leyva CB. Dolor agudo postoperatorio en el anciano. MediSur. 2013;11:443-449.

80. Velázquez GK. Deficiencias del tratamiento del dolor postoperatorio. Particularidades en el anciano. Rev Cubana de Anestesiol Reanim. 2012;11:57-65.

81. Cadavid-Puentes A, Bermúdez-Guerrero FJ, Giraldo-Salazar O, Muñoz-Zapata F, Otálvaro-Henao J, Ruíz-Sierra J, et al. Comparison of the effectiveness of fentanyl versus morphine for severe postoperative pain management. A randomized, double blind, clinical trial. Rev Colomb Anestesiol. 2017;45:100-107.

82. Orizondo PS, Caignet AM, Morúa DV, Salgado BY, Falcón GM. Analgesia postoperatoria con morfina intratecal en cirugía proctológica. Revista Cubana de Anestesiología y Reanimación. 2011;10:135-143.

83. Periañez CAH, Diaz MAC, Bonisson PLV, Simino GPR, Barbosa MH, Mattia AL. Relationship of anxiety and preoperative depression with post-operative pain. Texto \& Contexto - Enfermagem. 2020;29:e20180499.

84. Robleda G, Sillero-Sillero A, Puig T, Gich I, Baños JE. Influence of preoperative emotional state on postoperative pain following orthopedic and trauma surgery. Revista Latino-Americana de Enfermagem. 2014;22:785-791.

85. Vivas JP, Reyes E, Ríos F, Lozano R, Pérez JA, Plazas M. Measurement of satisfaction with anesthetic recovery in a high-complexity 
postanesthetic care unit: a patient perspective. Colombian Journal of Anesthesiology. 2018;46:279-285.

86. Eslava-Schmalbach J, Gaitán-Duarte H, Gómez-Restrepo C. Escala para medir la calidad de la recuperación postanestésica desde la perspectiva del usuario. Rev Salud Publica. 2006;8:52-62.

87. Wu CL, Naqibuddin M, Rowlingson AJ, Lietman SA, Jermyn RM, Fleisher LA. The effect of pain on health-related quality of life in the immediate postoperative period. Anesth Analg. 2003;97:1078-1085.

88. García-Andreu J. Manejo básico del dolor agudo y crónico. Anestesia en México. 2017;29:77-85.

89. Joshi G, Babatunde O. Consequences of inadequate postoperative pain relief and chronic persistent postoperative pain. Anesthesiology Clin N Am. 2005;23:21-36.

90. Wu CL, Rowlingson AJ, Partin AW, Kalish MA, Courpas GE, Walsh PC, et al. Correlation of post-operative pain to quality of recovery in the immediate post-operative period. Reg Anaesth Pain Med. 2005;30:516-522.

91. Tong D, Chung F, Wong D. Predictive factors in global and anesthesia satisfaction in ambulatory surgical patients. Anesthesiology. 1997;87:856-864.

92. Colvin LA, Power I. Pain management and quality in healthcare Best Pract Res Clin Anaesthesiol. 2001;15:633-653.

93. Perkins FM, Kehlet $\mathrm{H}$. Chronic pain as an outcome of surgery: a review of predictive factors. Anesthesiology. 2000;93:1123-1133.

94. Macrae WA. Chronic post-surgical pain: 10 years on. Br J Anaesth. 2008;101:77-86.
95. Quinde Cobos P. Dolor postoperatorio: factores de riesgo y abordaje. Medicina Legal de Costa Rica. 2017;34:254-264.

96. Kehlet H, Jensen TS, Woolf CJ. Persistent postsurgical pain: risk factors and prevention. Lancet. 2006;367:1618-1625.

97. Chinchilla HP, Baquero ZD, Guerrero NC, Bayter ME. Incidencia y factores asociados al dolor crónico postoperatorio en pacientes llevados a herniorrafia inguinal. Rev Colomb Anestesiol. 2017;45:291299.

98. Steeds CE. The anatomy and physiology of pain. Surg. 2013;31:49-53.

99. Katz J, Seltzer. Transition from acute to chronic postsurgical pain: risk factors and protective factors. Expert Rev Neurother. 2009;9:723-744.

100. Arboleda MF, Girón-Arango L, Peng PWH. Can Recent Chronic Pain Techniques Help With Acute Perioperative Pain? Curr Opin Anaesthesiol. 2019;32:661-667.

101. Walder B, Schafer M, Henzi I, Tramer MR. Efficacy and safety of patient-controlled opioid analgesia for acute postoperative pain. A quantitative systematic review. Acta Anaesthesiol Scand. 2001;45:795-804.

102. Bartels K, Mayes LM, Dingmann C, Bullard KJ, Hopfer CJ, Binswanger IA. Opioid Use and Storage Patterns by Patients after Hospital Discharge following Surgery. PLoS ONE. 2016;11:e0147972.

103. Mugabure Bujedo B, González Santos S, Tranque Bizueta I, Araujo López A, Torán García L. Manejo del dolor perioperatorio de los pacientes en tratamiento crónico con opioides. Revista de la Sociedad Española del Dolor. 2009;16:288-297. 